\title{
Ciclismo de Montaña como Motor del Desarrollo Sostenible del Turismo Local en Ecuador
}

\author{
Jesús A. Gómez ${ }^{(1)}$, Juan, M. Mantilla(2), Miguel, A. Posso ${ }^{(3)}$ y Ximena Maldonado(1) \\ (1) Universidad de Otavalo, Carrera de Derecho, Av. Sarances y los Pendoneros, Otavalo, Ecuador. \\ (e-mail: jgomez@uotavalo.edu.ec; xmaldonado@uotavalo.edu.ec) \\ (2) Universidad de Otavalo, Carrera Desarrollo Social y Cultural, Av. Sarances y los Pendoneros, Otavalo, \\ Ecuador. (e-mail: jmantilla@uotavalo.edu.ec) \\ (3) Universidad Técnica del Norte, Av. 17 de Julio 5-21, Ibarra, Ecuador. (e-mail: maposso@utn.edu.ec)
}

Recibido Feb. 6, 2018; Aceptado Abr. 10, 2018; Versión final Jun. 4, 2018, Publicado Oct. 2018

\begin{abstract}
Resumen
El objetivo principal de la investigación fue establecer los beneficios del ciclismo de montaña turístico para el desarrollo local sostenible en la ciudad de Ibarra de Ecuador. El tipo de investigación fue descriptiva y los instrumentos empleados fueron registro de campo mediante encuestas. El cuestionario dirigido a una muestra de 126 participantes de los 420 asistentes en la Vuelta al Imbabura del año 2015. La validación del método se realizó mediante el juicio de expertos seleccionados con el coeficiente de Conocimiento (Kc) y la confiabilidad interna mediante el coeficiente de Alfa de Cronbach del programa SPSS. Como resultado, se evidenció beneficios económicos, sociales, ambientales que influyeron en el desarrollo de la ciudad como uno de los destinos turísticos de Ecuador. Se concluyó que el ciclismo de montaña promovió los principios basados en la sustentabilidad favoreciendo el desarrollo local mediante actividades relacionadas con el turismo que genera este deporte.
\end{abstract}

Palabras clave: beneficios económicos; ciclismo de montaña; desarrollo local; turismo

\section{Mountain Biking for Sustainable Development of Local Tourism in Ecuador}

\begin{abstract}
The main objective of the research was to establish the benefits of touristic mountain biking for sustainable local development in the city of Ibarra in Ecuador. The study was based of quantitative type and the instrument used was field data gathered through a questionnaire. This questionnaire was applied to a sample of 126 people of the 420 participants of the Imbabura Circuit in the year 2015. The validation of the method was done through the judgement of selected experts with the Knowledge coefficient $(\mathrm{Kc})$ and the internal reliability was assessed using the Cronbach Alpha coefficient reported by the software SPSS. As a result, it was determined that the city obtained economic, social and environmental benefits that have influenced the development of the city to become one of the tourist destinations of Ecuador. It was concluded that mountain biking promoted principles based on sustainability that favored local development through activities related to tourism that this sport generates.
\end{abstract}

Keywords: economic benefits; mountain bike; local development; tourism 


\section{INTRODUCCIÓN}

El desarrollo local sustentable representa para los países en vías de desarrollo, una esperanza para mejorar el nivel de progreso en el urbanismo y la calidad de vida de los habitantes (Cobbinah, et al., 2015), y está orientado a generar estrategias relacionadas con el área social, turística, económica que provienen de los organismos públicos, privados para hacer un mejor uso racional de los recursos naturales. (Soto, et al., 2017; Pires et al., 2014). Así, el desarrollo del emprendimiento sostenible se fundamenta en la acción innovadora que permite mejorar el intercambio social entre las comunidades. Esto propicia un espacio de emprendimiento comercial en la localidad a través de las instituciones, organismos que suman esfuerzos para estandarizar indicadores que miden el desarrollo sostenible en diferentes áreas de la ciudad (Muñoz y Dimov, 2015). Entre ellos, el desarrollo local turístico, es considerado como una estrategia clave que contribuye con el desarrollo económico y el capital humano, convirtiéndose en una opción viable para el desarrollo turístico (Nunkoo, 2015), estableciendo enlaces de cooperación con agentes externos para capturar recursos humanos, técnicos y monetarios (Koukios et al., 2018). Por ello, (Sauvé et al., 2016), las dimensiones del desarrollo local, están estrechamente relacionadas con la economía, el medio ambiental y la protección ambiental. Esta última dimensión del medio ambiental (Belletti et al., 2017), la gestión de recursos de inversión individual o colectiva deben preservar y garantizar los recurso naturales de la localidad. Esta dimensión hace alusión a la conservación, el manejo responsable del medio ambiente a la hora de buscar la sostenibilidad, no solo es competencia de los entes gubernamentales como el estado, sino también involucra a otras instituciones como entidades privadas, organizaciones, grupos sociales que son responsables de contribuir con las soluciones del entorno y tecnologías que ocasionen menor impacto en la naturaleza.

(Reimer y Walter, 2013; Qian et al., 2017), mediante estudios de proyectos ecoturísticos dirigidos a la comunidades en Camboya y la China se puede impulsar la protección del medio y el desarrollo turístico. Al igual (Burivalova, et al., 2017) es necesario crear iniciativas que aseguren la sostenibilidad de los recursos naturales a fin de promover la conservación del medio ambiente. En efecto, las localidades deben tomar como eje fundamental la preservación del medio ambiente además del bienestar social y la prosperidad económica en el ámbitolocal. De ahí (Muñoz, 2009) ayudan a preservar el medio ambiente es el ciclismo de montaña, considerado como un importante generador de viajes turísticos facilita el aprovechando para crear toda una industria de servicios, comercio que benefician a las localidades, habitantes, desarrolla el turismo como una de sus principales actividades económicas, enfocado principalmente en el aprovechamiento sostenible de la naturaleza, el turismo activo, de aventura, ecoturismo y el turismo sostenible. (Pickering y Rossi, 2016) la bicicleta de montaña posee una apreciación positiva hacia las actividades recreativas, no motorizadas sustentada en valores ecocéntrico que ayudan a la conservación del medio ambiente para el desarrollo sostenible. Sin embargo, uno de los tipos más recientes de bicicleta, es la de montaña (Pickering, et al., 2016; Hagen y Boyes, 2016) generan mayor comodidad para el transporte se ha convertido en una herramienta recreativa y su utilización está en constante crecimiento, permitiendo que las personas puedan aprovechar y llegar en bicicleta a escenarios naturales que no eran factibles décadas atrás. (Zhang et al., 2015), este vehículo sostenible se ha convertido en una herramienta para conocer sitios turísticos con mayor facilidad, así entonces se puede aprovechar estas condiciones para desarrollar el turismo de ciclismo de montaña en destinos con escenarios geográficas adecuadas.

En el caso de Ecuador, (Ministerio de Turismo, 2018), ha demostrado que uno de los principales objetivos de la nación Ecuatoriana es el desarrollo turístico, se encuentra entre las cinco principales actividades económicas del país, al igual el (Boletín Estadísticas Turísticas del año, 2012-2016) durante el mes de enero del 2015, se registró un crecimiento en las llegadas a Ecuador en el orden del $11 \%$ con respecto al mismo mes del año anterior. En el cantón lbarra, el desarrollo del turismo ha sido siempre uno de los objetivos de las administraciones públicas y del sector privado. En consecuencia, el diario (El Norte, 2016), publicó la Vuelta al Imbabura 2015, séptima edición que se realizó en la ciudad de Ibarra, provincia Imbabura, Ecuador, la misma tuyo como propósito desarrollar la competencia de ciclismo de montaña a fin de promover el desarrollo local sostenible de la zona, empleando el turismo de montaña que bordeó el coloso volcán "Imbabura". Fue una competencia de punto a punto de $40 \mathrm{kms}$ de distancia que recorrió caminos rodeado de paisajes naturales. Partió desde el Coliseo de Deportes "Francisco Páez" en la ciudad de Otavalo, recorrió impresionantes paisajes atravesando las comunidades de la Compañía, Camuendo, Loma Cunga, Abatag, Cochaloma, El Abra, Chirihuasi, Rumipamba, El Yaguachi, La Esperanza, San Juan, Santa Rosa del Tejar, San Eduardo, Las Malvinas, La Campiña y terminó en el bosque protector Loma de Guayabillas de la ciudad de Ibarra. Dicha ruta define al ciclismo de montaña una competencia individual que contribuye con la formación integral del ser humano, fomenta la aptitud ambientalista, principios de sostenibilidad y de protección al medio ambiente, (Sportbike, 2018; La Hora, 2017; Angelkova et al., 2012). La geomorfología de la Vuelta consiste en circunvalar el volcán que comprende una distancia total de $40 \mathrm{Kms}$ de distancia, pasando por distantos pueblos de la provincia tal como se evidencia en la Figura 1. 


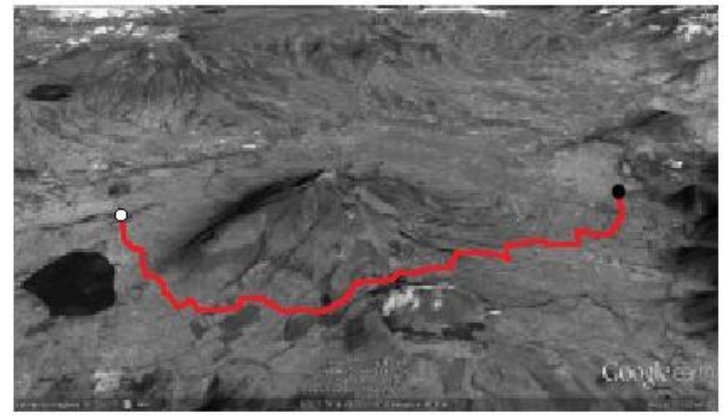

Fig. 1: Mapa Vuelta Imbabura 2015, Línea de recorrdio ------- punto de partida $\mathbf{O}$ y punto de llegada

El punto de partida de la competencia es el Coliseo Deportivo Francisco Páez ubicado en la ciudad de Otavalo a una altura de 2300 m.s.n.m, luego el recorrido sigue por zonas rurales como la Compañía, Cochaloma, La Esperanza, El Yaguachi, Santa Rosa del Tejar, cruza el sector El Abra con 3430 m.s.n.m, hasta llegar a la ciudad de lbarra, altura de 2220 m.s.n.m, (El Norte, 2018; la Hora, 2017). Para el (Norte, 2017; Sportbike, 2018) la competencia estuvo organizada por Carlos Revelo, su misión es promover la practica del ciclismo de montaña aprovechando las bondades del paisaje turístico de la zona a fin de auspiciar la belleza de la ciudad Ibarra y la provincia. No obstante, (Nesbitt et al., 2017; Manhas et al., 2016) el valor del servicio ecoturísticos urbano constituye un enfoque cultural clave para el bienestar de las personas y el desarrollo del destino turístico fortalece la imagen de marca. La ciudad de lbarra presenta pocas oportunidades que impulsen el desarrollo local, carece de una infraestructura de negocios, servicios turísticos, espacios especializados para el nuevo visitante, fuentes de trabajo y gasto turístico. Por ello, el ciclismo de montaña (Alarcón et al., 2016) señala que es una oportunidad que fortalece la oferta turística, genera oportunidades de emprendimiento, conocimiento para planificar en mejor manera el crecimiento sostenible de la ciudad de Ibarra. El ciclismo de montaña (Pickering y Rossi, 2016), es una actividad que fortalece la oferta turística, un mayor incrementando económico, desarrollo de eventos y competencias en la ciudad. En el marco de estas observaciones se presenta como problema científico, el siguiente ¿Cómo beneficiar a través del ciclismo de montaña el desarrollo local sostenible de la ciudad de Ibarra, la provincia de Imbabura, Ecuador?

Según (Taher et al., 2015), el turismo de montaña tiene como característica principal la accesibiidad a los paisajes, es una herramienta de crecimiento para el ser humano debido al aprendizaje, la participación y la interacción con nuevos ambientes naturales. También, el turismo es considerado como un fenómeno social, cultural, económico que está vinculado con el movimiento de la persona hacia otros lugares distintos al sitio donde habita que ocurre por motivos personales, de negocios o profesionales. A este tipo de personas se le denomina visitantes, viajeros, turistas que se trasladan a un espacio determinado, implica a su vez un gasto turístico a fin de favorecer el desarrollo socio económico local mediante acciones sostenibles que involucra personas, sociedad, entes públicos y privados. Por otra parte, la relación bicicleta y el turismo según (Fishman et al., 2014; Hagen y Boyes 2016) el uso de la bicicleta es un transporte sostenible, y seguro que pasa de la necesidad recreativa del habitante hasta considerase como un medio de movilización. Es una actividad rentable y beneficiosa para la calidad de vida de las personas, al mismo tiempo, se ajusta a un plan de desarrollo integral, sustentable urbano que evita problemas futuros, tales como congestionamiento de las vías, autopistas, aglomeramiento vehicular y disminuye la contaminación ambiental.

De estas consideraciones, se abordó las variables a nivel teórico que permitieron fundamentar el estudio y la problemática seleccionada tales como: los recursos y potencialidades que posee la ciudad de Ibarra, la relación que existe entre el ciclismo de montaña, el turismo, el desarrollo sostenible y sus beneficios. Seguidamente, el objetivo general del estudio fue establecer los beneficios del ciclismo de montaña y el turismo en el desarrollo local sostenible de la ciudad de Ibarra, durante la Vuelta al Imbabura 2015 en la provincia de Imbabura, Ecuador. Identificar los recursos y potencialidades que posee la ciudad de Ibarra para la promoción del desarrollo local sostenible. Describir la relación del ciclismo de montaña y el turismo para el desarrollo local sostenible de la ciudad objeto de estudio y por ultimo establecer los beneficios del ciclismo de montaña para el desarrollo local sostenible de la ciudad de lbarra.

\section{MATERIALES Y MÉTODOS}

La metodología utilizada fue investigación de tipo descriptivo (Barrera, 2007), esta consistió en conocer la situación exacta sobre el ciclismo durante la Vuelta al Imbabura 2015, el desarrollo local sostenible y el turismo en la ciudad de Ibarra. El mapeo de la Vuelta se inició desde las instalaciones del Coliseo Deportivo Francisco Páez, realizó un recorrido de 40 KMs alrededor del volcán hasta llegar a la ciudad de Ibarra (EI Norte, 2016). De acuerdo con las consideraciones éticas la investigación se fundamentó en el principio de beneficencia (Hoyos, 2000), cuyo fin es hacer el bien a las personas, disminuir cualquier tipo de riesgo y conseguir el 
máximo beneficio común de las personas. Al evento asistieron (420) personas consideradas como población de estudio, el muestreo escogido fue de tipo probabilístico o aleatorio, (Hernández et al., 2014), de acuerdo con el nivel de confianza, el tamaño de la muestra quedó conformada por (126) personas. El instrumento aplicado a la muestra fue el cuestionario, diseñado por los autores de la investigación, estructurado en (18) ítems relacionados con el ciclismo de montaña y el turismo (Hernández et al., 2014). Se utilizó como pre test para garantizar la claridad y confiabilidad de las preguntas con previo consentimiento de las personas enmarcado en el principio ético de beneficencia. La validez de contenido del cuestionario se determinó a través de la técnica de juicio de experto, integrado por (03) profesionales seleccionados con el coeficiente de conocimiento $(\mathrm{Kc})$ y la confiabilidad interna con el coeficiente de Alfa de Cronbach de análisis univariado del programa estadístico SPSS.

\section{RESULTADOS}

La primera etapa de resultados, se corresponde con el cuestionario aplicado a la muestra, describió la relación del ciclismo de montaña y el turismo, así como también los beneficios durante la Vuelta al Imbabura 2015, como se evidencia en la tabla 1:

Tabla 1: Distribución de la opinión de los encuestados sobre el ciclismo de montaña y el turismo

\begin{tabular}{|c|c|c|c|c|c|c|c|}
\hline \multirow{2}{*}{$N^{\circ}$} & \multirow{2}{*}{ Dimensión ciclismo de montaña y el turismo } & \multicolumn{2}{|c|}{$\mathrm{Si}$} & \multicolumn{2}{|c|}{ No } & \multicolumn{2}{|c|}{ TOTAL } \\
\hline & & $F$ & $\%$ & $F$ & $\%$ & $F$ & $\%$ \\
\hline 1 & $\begin{array}{l}\text { ¿El ciclismo de montaña es un deporte que } \\
\text { promueve el turismo local? }\end{array}$ & 124 & 98,41 & 2 & 1,59 & 126 & 100,00 \\
\hline 2 & $\begin{array}{l}\text { ¿El ciclismo de montaña es una práctica lúdica y } \\
\text { recreativa que contribuye con la saludo el medio } \\
\text { ambiente? }\end{array}$ & 126 & 100,00 & 0 & 0,00 & 126 & 100,00 \\
\hline 3 & $\begin{array}{l}\text { ¿Mediante el ciclismo de montaña se puede dar a } \\
\text { conocer las potencialidades y los recursos } \\
\text { turísticos de la zona? }\end{array}$ & 109 & 86,51 & 17 & 13,49 & 126 & 100,00 \\
\hline 4 & $\begin{array}{l}\text { ¿La bicicleta es un medio de transporte ecológico } \\
\text { sostenible? }\end{array}$ & 126 & 100,00 & 0 & 0,00 & 126 & 100,00 \\
\hline
\end{tabular}

La tabla 1, referente a las respuesta de los encuestados sobre el ciclismo de montaña y el turismo, se logró observar en el ítem 1 , el noventa y ocho por ciento coma cuarenta y uno $(98,41 \%)$ de los ciclistas, señaló el ciclismo de montaña es un deporte que promueve la actividad turística, solo uno coma cincuenta y nueve ( 1 , $59 \%)$ manifestó que no. A partir de esta información, la mayoría de los participantes afirman que a través de este deporte representa una alternativa para promocionar la actividad turística como medio para lograr el desarrollo sustentable de la ciudad. El ítem 2, reflejó cien por ciento (100\%) de los participantes coinciden que el ciclismo de montaña es una actividad lúdica, recreativa que fortalece la salud de las personas y al medio ambiente. El ítem 3, el ochenta y seis coma cincuenta y uno por ciento $(86,51 \%)$ de los ciclistas opinaron este tipo de práctica permite dar a conocer, promocionar las potencialidades, recursos turísticos que posee la provincia, solo un trece como cuarenta y nueve $(13,49 \%)$ expresó que no. Por último, el ítem 4 , el cien por ciento (100\%) expresó que si está de acuerdo la bicicleta es un medio de transporte ecológico sostenible que ayuda con la protección del medio ambiente y la sostenibilidad de la ciudad de Ibarra.

Tabla 2: Distribución de la opinión de los encuaestados sobre los beneficios del ciclismo de montaña

\begin{tabular}{cccccccc}
\hline \multirow{2}{*}{$N^{\circ} \quad$ Dimensión beneficios del ciclismo de montaña } & \multicolumn{2}{c}{$S i$} & \multicolumn{2}{c}{ No } & \multicolumn{2}{c}{ TOTAL } \\
\cline { 3 - 7 } & $F$ & $\%$ & $F$ & $\%$ & $F$ & $\%$ \\
\hline 5 & $\begin{array}{l}\text { ¿El ciclismo de montaña fomenta el respecto por la } \\
\text { naturaleza? }\end{array}$ & 126 & 100,00 & 0 & 0,00 & 126 & 100,00 \\
$\quad \begin{array}{l}\text { iEl ciclismo de montaña puede ayudarte a descubrir } \\
\text { lugares únicos de difícil acceso? }\end{array}$ & 121 & 96,03 & 5 & 3,97 & 126 & 100,00 \\
$\quad \begin{array}{l}\text { iEl uso de la bicicleta ocasiona algún tipo de } \\
\text { contaminación ambiental? }\end{array}$ & 6 & 4,76 & 120 & 95,24 & 126 & 100,00 \\
$\quad \begin{array}{l}\text { LLa práctica del ciclismo de montaña contribuye con } \\
\text { la salud integral y el desarrollo sustentable de la } \\
\text { ciudad? }\end{array}$ & 126 & 100,00 & 0 & 0,00 & 126 & 100,00 \\
\hline
\end{tabular}

En cuanto a la Tabla 2, referente a las opiniones de los encuestados sobre el beneficio del ciclismo de montaña, se logró constatar en el ítem 5, el cien por ciento (100\%) de los ciclistas, expresaron este tipo de 
deporte ayuda a fomentar el respeto por la madre naturaleza. El ítem 6, evidenció un noventa y seis coma cero tres por ciento $(96,03 \%)$ de los participantes concuerdan que el ciclismo de montaña facilita a las personas llegar a lugares de difícil acceso que poseen un paisaje extraordinario de la zona. El ítem 7, el noventa y cinco coma veinte cuatro por ciento $(95,24 \%)$ de los participantes señalaron el uso de la bicicleta no genera ningún tipo de contaminación ambiental. El ítems 8, el cien por ciento (100\%) expresó la práctica de ciclismo de montaña favorece la salud integral y el desarrollo sustentable de la ciudad de lbarra.

Tabla 3: Utilización de servicios turísticos complementarios

\begin{tabular}{lcc}
\hline & \multicolumn{1}{c}{$\begin{array}{l}\text { Ítems 9. } \\
\text { ¿Cuáles de estos servicios turísticos complementarios } \\
\text { utilizas más en la ciudad de Ibarra durante la Vuelta al } \\
\text { Imbabura 2015? }\end{array}$} & Servicios turísticos complementarios \\
\cline { 2 - 3 } SPA & $F$ & $\%$ \\
Balnearios & 45 & 35,71 \\
Actividades de ocio & 60 & 47,62 \\
Transporte turístico & 10 & 7,94 \\
Guía turístico & 20 & 15,87 \\
\hline Total & 36 & 28,57 \\
\hline
\end{tabular}

Los servicios turísticos complementarios más utilizados durante la Vuelta al Imbabura 2015, de los encuestados, 45 personas, (35,71\%) acudieron a los SPS, Balnearios 60 participantes, $(47,62 \%)$. El mayor número de los ciclistas acudieron a estos servicios es porque la carrera es una actividad deportiva de entretenimiento, donde los competidores optan por este tipo de servicio para el descanso, la relajación y actividades de ocio, (7,94\%). Seguidamente 20 personas, $(15,87 \%)$ de todos los participantes utilizaron el transporte turístico, la mayoría utilizó el transporte particular y público. Guías turísticos, 36 personas, $(28,57 \%)$ utilizó este tipo de apoyo que acudió a lugares turísticos de la ciudad. Seguidamente se presenta a continuación la Tabla 4. Lugar de preferencia para consumir alimentos en Ibarra relacionada con el ítems 10.

Tabla 4: Lugar de preferencia para consumir alimentos en Ibarra

\begin{tabular}{|c|c|c|}
\hline \multirow{2}{*}{$\begin{array}{c}\text { Ítems } 10 . \\
\text { ¿Cuál es el lugar de preferencia para consumir alimentos } \\
\text { en lbarra? }\end{array}$} & \multicolumn{2}{|c|}{ Lugar de preferencia para consumir alimentos en Ibarra } \\
\hline & $F$ & $\%$ \\
\hline Lugar de alojamiento & 7 & 5.5 \\
\hline Comida chatarra & 10 & 7.9 \\
\hline Patios de comidas & 17 & 13.5 \\
\hline Restaurantes de la ciudad & 92 & 73.0 \\
\hline Total & 126 & 100 \\
\hline
\end{tabular}

El lugar de preferencia para el consumo de alimentos en Ibarra El 73,0\% de los competidores prefirieron los restaurantes de la ciudad; seguido por el $13,5 \%$ que van a patios de comidas, el $7.9 \%$ optaron por consumir comida chatarra, es decir comida de la calle, el $5.5 \%$ consumieron alimentos en el lugar de alojamiento. Estos lugares de preferencia fueron los sitios turísticos con mayor concurrencia por parte del participante en la ciudad durante la Vuelta al Imbabura 2015.

Tabla 5: El ciclismo de montaña y el desarrollo local sostenible

\begin{tabular}{|c|c|c|}
\hline \multirow{2}{*}{$\begin{array}{c}\text { Ítems } 11 . \\
\text { ¿Selecciones los elementos que están relacionados con } \\
\text { el desarrollo local sostenible? }\end{array}$} & \multicolumn{2}{|c|}{ Lugar de preferencia para consumir alimentos en Ibarra } \\
\hline & $F$ & $\%$ \\
\hline Ecoturismo & 10 & 7,94 \\
\hline Sostenibilidad. & 15 & 11,90 \\
\hline Actividad recreativa. & 6 & 4,76 \\
\hline Protección del medio ambiente. & 10 & 7,94 \\
\hline Políticas sostenibles locales & 45 & 35,71 \\
\hline $\begin{array}{l}\text { Servicios turísticos, trasporte, hoteles y agencias de } \\
\text { viajes. }\end{array}$ & 40 & 31,75 \\
\hline Total & 126 & 100 \\
\hline
\end{tabular}


La selección de los elementos relacionados con el desarrollo local sostenible, se constató el $(35,71 \%)$ de los competidores expresaron que las políticas sostenibles juegan un papel importante para el desarrollo sostenible de la ciudad. El (31,75\%) de los encuestados, opinaron los servicios turísticos, trasporte, hoteles, y agencias de viajes representan el gasto turístico que contribuye con el desarrollo local de la ciudad de lbarra; Seguido del $(11,90 \%)$ manifestaron que el principio de sostenibilidad garantiza la racionalidad y la promoción de los recursos naturales como patrimonios turísticos.

Tabla 6: Plan de Desarrollo del Cantón Ibarra “Turismo" 2015

\begin{tabular}{|c|c|c|}
\hline \multirow{2}{*}{ Tipos de Establecimientos } & \multicolumn{2}{|c|}{ Plan de Desarrollo del Cantón Ibarra "Turismo" 2015} \\
\hline & $F$ & $\%$ \\
\hline Agencias de viajes & 16 & 5,84 \\
\hline Alojamiento & 62 & 22,63 \\
\hline Alimentos y bebidas & 193 & 70,44 \\
\hline Transporte & 3 & 1,09 \\
\hline Total & 274 & 100,00 \\
\hline
\end{tabular}

La Tabla 6. Plan de Desarrollo del Cantón Ibarra "Turismo" 2015, logró evidenciar la presencia de 16 Agencias de Viajes, representó un 5,84\%, en cuanto al alojamiento existen 62 instalaciones, (22,63\%), establecimiento de alimentos y bebidas, 193, representó $(70,44 \%$,$) líneas de trasporte 3, (1, 09 \%)$. En cuanto al trasporte sólo contó con tres (03) empresas registradas legalmente con capacidad limitada para 360 personas.

La segunda etapa de resultados, se enmarcó en el instrumento registro de campo, identificó distintos recursos y potencialidades relacionados con la ciudad de Ibarra. La provincia cuenta con diferentes establecimientos como atractivos turísticos que impulsan el desarrollo sustentable, tal como se evidencia en la siguiente tabla:

Tabla 7: Principales atractivos turísticos del cantón Ibarra

\begin{tabular}{|c|c|}
\hline Atractivos turísticos & Descripción \\
\hline $\begin{array}{l}\text { Laguna de } \\
\text { Yahuarcocha }\end{array}$ & $\begin{array}{l}\text { Es uno de los puntos hídricos más importantes del norte del país ya que posee un encanto } \\
\text { único por su biodiversidad, paisaje, se pueden realizar actividades. }\end{array}$ \\
\hline Automovilismo & $\begin{array}{l}\text { La laguna cuenta con una pista automovilística donde se realizan eventos nacionales e } \\
\text { internacionales. }\end{array}$ \\
\hline Parapente & $\begin{array}{l}\text { Este deporte de aventura se realiza de manera frecuente en la cuenca de la laguna y en } \\
\text { ocasiones se han hecho competencias de carácter nacional e internacional }\end{array}$ \\
\hline Muelle & $\begin{array}{l}\text { Está ubicado al noroccidente de la entrada principal de la laguna ofreciendo como principal } \\
\text { actividad paseos en bote a remo y lanchas a motor. También se puede encontrar una } \\
\text { discoteca y un restaurant. }\end{array}$ \\
\hline Gastronomía & $\begin{array}{l}\text { La oferta gastronómica del cantón lbarra es amplia pero está principalmente representada } \\
\text { por los típicos helados de paila, las nogadas y el arrope. }\end{array}$ \\
\hline Música & La banda mocha y la bomba forman parte del patrimonio intangible del cantón Ibarra. \\
\hline Ruta del Tren & $\begin{array}{l}\text { lbarra - Salinas es una ruta que recorre por hermosos paisajes naturales que adornan la } \\
\text { vía, en la llegada a Salinas la comunidad deleita a los visitantes con baile, música y cultura. }\end{array}$ \\
\hline $\begin{array}{l}\text { Artesanía en } \\
\text { Zuleta }\end{array}$ & $\begin{array}{l}\text { Esta comunidad es muy reconocida por las artesanía que elaboran sus mujeres y se ha } \\
\text { convertido en uno de los destinos preferidos para los turistas. }\end{array}$ \\
\hline $\begin{array}{l}\text { Deportes de } \\
\text { Aventura }\end{array}$ & $\begin{array}{l}\text { En el cantón lbarra se pueden practicar varios tipos de deportes de aventura como rafting, } \\
\text { canyoning, puenting, escalada, MTB y parapente }\end{array}$ \\
\hline $\begin{array}{l}\text { Artesanías de San } \\
\text { Antonio de } \\
\text { lbarra }\end{array}$ & $\begin{array}{l}\text { Reconocidos por sus tallados en madera pero también realizan tejidos, trabajos en cerámica } \\
\text { piedra. Imágenes religiosas costumbristas son las de más demanda tienen por parte de los } \\
\text { turistas. }\end{array}$ \\
\hline
\end{tabular}

La ciudad de lbarra conserva otros establecimientos que se caracterizan por un potencial atractivo turístico e impulsan el desarrollo sustentable de la provincia, entre ellos se menciona la laguna de Yahuarcocha, Parapente, El Muelle, Ruta del Tren, son destinos turísticos para el ciclismo de montaña por sus condiciones geográficas, paisajes y escenarios climáticos, permiten la promoción de otras actividades vinculadas con el ciclismo y el diseño de rutas para realizar competencias nacionales e internacionales. 


\section{DISCUSIÓN}

La ciudad de lbarra, se ubica en un cantón que dispone de recursos naturales con un gran potencial turístico que permite promover el desarrollo sustentable de la localidad, tal es el caso, la laguna de Yahuarcocha calificada como de los destinos turísticos para el ciclismo de montaña por sus características geográficas, circunstancias climáticas que facilitan el desarrollo de actividades vinculadas con otros deportes, automovilismo, parapente, canyoning, deportes de aventura (Cuascota et., al 2017). Así, los recursos y potencialidades turísticas de la ciudad de Ibarra, combinado con el ciclismo de montaña en la Vuelta la Imbabura, impulsan el desarrollo local debido a la estrecha relación con la sostenibilidad, particularmente con características que aseguran las necesidades del presente sin perjudicar las próximas generaciones, es decir generar nuevos recursos, desarrollo económico con participación de actores locales. Además la sostenibilidad se fundamenta en principios y condicionantes esenciales para el desarrollo local, tales como: el interés en la naturaleza que contribuye con la conservación, dependencia en áreas naturales protegidas, la disminución del impacto ambiental y una mayor conciencia por la madre naturaleza (Taher et al., 2015). De este modo, la presente investigación verificó que el consumo de servicios turísticos generados por el desarrollo del ciclismo de montaña puede mejorar significativamente la ciudad, ampliar los servicios turísticos, espacios especializados para los visitantes extranjeros, el trasporte, disponer de espacios para el ocio, la diversión y una mejorar la infraestructura hotelera para recibir a los visitantes. De la misma manera, el mapeo empleado durante la Vuelta al Imbabura 2015, se caracterizó por obtener una geomorfología dinámica y extraordinaria, debido a sus diferentes paisajes, vistas, pueblos desde la zona de partida hasta llegar a la ciudad de Ibarra (El Norte, 2018; la Hora, 2017).

A nivel internacional, países como Portugal, Australia, República Checa, Sur África, el ciclismo de montaña es considerado como un medio para el desarrollo sostenible de las zonas rurales, ayuda a la creación de políticas destinadas a administrar los sitios como destinos turísticos, (Boori et al., 2015; Du Preez y Lee, 2016; Hardiman y Burgin 2011; Kastenholz et al., 2012). Este hecho, se convierte en un factor motivante para la provincia, generó nuevos emprendimientos en el área del turismo, facilitó el aprovechamiento de la infraestructura local, uso de la red hotelera, trasporte beneficiando el sector empresarial y la industria turística, asi mismo, realizar investigaciones futuras que justifiquen este tipo de necesidad que contribuyan con el desarrollo sostenible de la ciudad de lbarra. Además despertó el interés en este sector por ofertar mejor servicio turístico, realizar nuevos evento como son: tours en bicicleta por lugares patrimoniales, tours gastronómicos y recorrido de montaña como atractivos turísticos enmarcado en el desarrollo sustentable de la provincia, (El Norte, 2016; Sebele, 2010). Igual manera, la ruta seleccionada durante la Vuelta, orientó al ciclista como competidor a considerar el turismo como una actividad de gran impacto para el desarrollo sustentable de la ciudad, el uso de la bicicleta fue el medio de transporte ecológico que despertó el interés por valorar el medio ambiente, la conservación de la naturaleza que contribuyó con la conservación de los recursos naturales y la sostenibilidad (Angelkova et al., 2012; O'brien et al., 2014).

El turismo alternativo basado en el beneficio del ciclicmo de montaña permitió a nivel ambiental, el contacto con la madre naturaleza ayudó a la salud física y mental de los participantes, contribuyó con la protección del ecosistema, concienciar a las personas sobre la importancia de los recursos naturales, evitar la tala, la quema que destruyen el medio ambiente, el ciclismo de montaña en un deporte ecológico es decir que no ocasiona daños al ecosistema, (Snyman, 2012). En lo social, el ciclismo es una actividad deportiva de turismo de aventura beneficia significativamente a la economía, tiene un gran potencial por sus condiciones favorables en su ejecución y equipamiento módico, promueve el mejoramiento de las relaciones interpersonales mediante el contacto con espacios naturales, senderos como destinos turísticos que ayudó ciclista al crecimiento personal y mental como ser humano (Soto, et al., 2017). En efecto, el ciclismo generó un beneficio significativo y sostenible en el desarrollo local, permitió la producción de fuentes de empleo, industria inversión pública, privada, aumento de los flujos turísticos, reposicionamiento de la ciudad como destino turístico, reproyección de la imagen de la ciudad y el desarrollo comunitario. Por ejemplo, la competencia la "Vuelta al Imbabura" está posicionada como una competencia de alto nivel técnico, cuya ruta dispone de un paisaje natural extraordinario, este evento es aprovechado para mejorar la imagen de lbarra como destino turístico de deporte, aventura y naturaleza para el desarrollo sostenible (Jaafar et al., 2011). Por tanto, la ciudad de Ibarra es vista como unos de los destinos preferidos de Ecuador por su potencial turístico patrimonial, artesanal, histórico y cultural de América Latina como se evidencia en la figura 2.

En la figura 2, denominada relación del turismo con el desarrollo sostenible, presenta los elementos esenciales que promueven el progreso local sostenible de la ciudad. Para (Castellani y Sala, 2010) estos factores basados en el turismo como es la aventura, el ecoturismo, responsabilidad, facilitan la creación de políticas destinadas a fomentar nuevas formas de emprendimiento, negocios, fuentes de empleo, asi como también proteger los paisajes naturales utilizando como medio la actividad turística y los recursos naturales de la zona. (Snyman, 2012), la combinación del turismo con el ciclismo de montaña como actividad económica conduce a la creación de empresas mixtas de ecoturismo entre el sector rural y urbano, con el propósito de mejorar la 
calidad de vida, solución a la pobreza, beneficios de bienestar social, educativo, ambiental y el progreso económico de los habitantes de la provincia. De este modo, la Vuelta al Imbabura, se convierte en el medio que facilita la gestión de los recursos naturales para el desarrollo económico, sostenible de las zonas rurales, la ruta seleccionada cada año beneficia directamente a los pueblos, comunidades, habitantes debido a la concurrencia de empresas turísticas, autoridades locales, visitantes que contribuyen con a calidad de vida y la protección del medio ambiente, (Angelkova et al., 2012; Sportbike, 2018). Por ello, en cada edición de la vuelta, el diseño de las rutas ciclísticas es producto del estudio técnico entre las autoridades, incluye principalmente las condiciones geomorfológicas de la zona en cada cantón de la provincia, así como también, las oportunidades del turismo de aventura, recreación, ecoturismo, turismo rural, convencional y alternativo (Ministerio de Turismo, 2018).

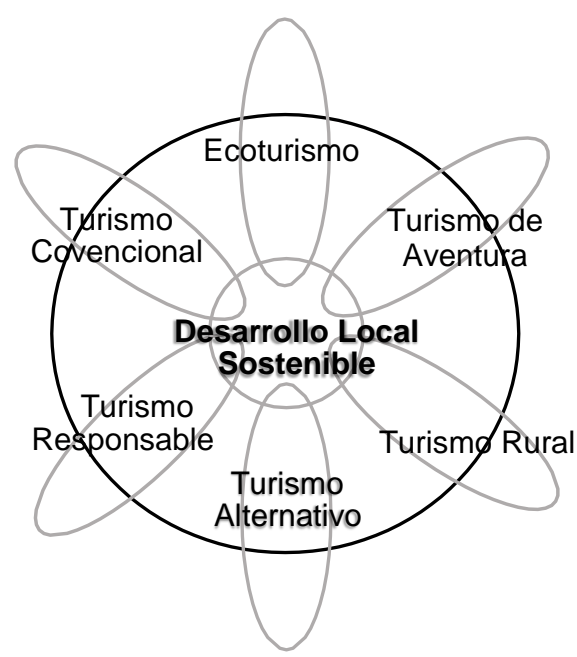

Fig. 2: Relación del turismo con el desarrollo sostenible.

\section{CONCLUSIONES}

El ciclismo de montaña generó beneficios significativos para la ciudad de Ibarra, en lo social, cultural y económico. Posicionó la imagen en nuevos mercados internacionales debido a su potencial turístico, permitiendo a la ciudad ofertar nuevos evento como son: tours en bicicleta por lugares patrimoniales, gastronómicos, recorrido de montaña como atractivos turísticos enmarcado en el desarrollo sustentable para la provincia.

La Vuelta al Imbabura, se establece como un icono turístico de referencia para otros países del mundo. Tal es el caso de Portugal, Australia, República Checa, Sur África, emplean el ciclismo de montaña como base para el desarrollo sostenible de las zonas rurales. Por ello, se convierte en un factor motivante para la creación de políticas destinadas a nuevos emprendimientos en el área del turismo, el aprovechamiento de la infraestructura local, el sector empresarial y la industria turística.

El mapeo empleado durante la Vuelta al Imbabura 2015, se caracterizó por obtener una geomorfología dinámica y extraordinaria por sus diferentes paisajes. La ciudad de Ibarra es vista como unos de los destinos preferidos de Ecuador por su exuberante patrimonio artesanal, histórico y cultural de América Latina. La ruta empleada, condujo al ciclista de montaña a valorar el turismo como una actividad que favorece el desarrollo sustentable, de ahí la bicicleta fue el medio de transporte ecológico que despertó el interés por proteger el medio ambiente, la naturaleza y conservar los recursos naturales de la zona.

\section{REFERENCIAS}

Alarcón-Aldana, A. C., J. Urrutia-Pinilla y M. Callejas-Cuervo, Aplicación Móvil para la Administración de Variables Físicas en Ciclismo al Aire Libre, doi: 10.4067/S0718-07642016000400019, Información Tecnológica, 27(4), 175-182 (2016)

Angelkova, T.C., Z. Koteski Jakovlev e E. Mitrevska, Sustainability and competitiveness of tourism, doi: 10.1016/j.sbspro.2012.05.023, Procedia-Social and Behavioral Sciences, 44, 221-227 (2012)

Barrera, M., Modelos Epistémicos en Educación e Investigación, 4aㅡ Ed., 23-42, Sypal, Quirón Ediciones, Caracas, Venezuela (2012)

Belletti. G., A. Marescotti y J. Touzard, Geographical indications, public goods, and sustainable development: The roles of actors' strategies and public policies, doi: 10.1016/j.worlddev.2015.05.004, World Development, 98, 45-57 (2017)

Boletín Estadísticas Turísticas del año, 2012-2016 (2018) 
Boori, M.S., V. Voženílek y K. Choudhary, Land use/cover disturbance due to tourism in Jeseníky Mountain, Czech Republic: A remote sensing and GIS based approach, doi: 10.1016/j.ejrs.2014.12.002, The Egyptian Journal of Remote Sensing and Space Science, 18(1), 17-26 (2015)

Burivalova, Z., F. Hua, L. Koh, C. Garcia y F. Putz, Critical comparison of conventional, certified, and community management of tropical forests for timber in terms of environmental, economic, and social variables, doi: 10.1111/conl.12244, Conservation Letters, 10(1), 4-14 (2017)

Castellani, V. y S. Sala, Sustainable performance index for tourism policy development. Tourism management, doi: 10.1016/j.tourman.2009.10.001, 31(6), 871-880 (2010)

Cobbinah, P. B., M. O. Erdiaw-Kwasie y P. Amoateng, Rethinking sustainable development within the framework of poverty and urbanisation in developing countries, doi:10.1016/j.envdev.2014.11.001, Environmental Development, 13, 18-32 (2015)

Du Preez. M. y D. E. Lee, The economic value of the Trans Baviaans mountain biking event in the Baviaanskloof MegaReserve, Eastern Cape, South Africa: A travel cost analysis using count data models, doi: 10.1016/j.jort.2016.07.003, Journal of Outdoor Recreation and Tourism, 15, 47-54 (2016)

El Norte, El ciclismo toma fuerza en Imbabura (2016)

El Norte, Vuelta al Imbabura con sello carchense, Ibarra Ecuador (2018)

Fishman, E., S. Washington y N. Haworth, Bike share's impact on car use: evidence from the United States, Great Britain, and Australia, doi: 10.1016/j.trd.2014.05.013, Transportation Research, Transport and Environment, 31, 13-20 (2014)

Ghasemi, M. y A. HamzahAn, Investigation of the appropriateness of tourism development paradigms in rural areas from main tourism stakeholders' point of view, doi: 10.1016/j.sbspro.2014.07.269, Procedia-Social and Behavioral Sciences, 144, 15-24 (2014)

Hagen, S. y M. Boyes, Affective ride experiences on mountain bike terrain, doi: 10.1016/j.jort.2016.07.006, Journal of Outdoor Recreation and Tourism, 15, 89-98 (2016)

Hardiman, N. y S. Burgin, Canyoning adventure recreation in the Blue Mountains World Heritage Area Australia, doi: 10.1016/j.tourman.2011.01.002, The canyoners and canyoning trends over the last decade, Tourism Management, 32(6), 1324-1331 (2011)

Hernández, R., C. Fernández y P. Baptista, Metodología de la Investigación, 6ª Ed., 270-299, Mc Graw Hill, D.F., México (2014) Hoyos, J.G.O. Principios éticos de la investigación en seres humanos y en animales, ISSN: 0025-768, Rev. Medicina, 60(2), 255-258 (2000)

Jaafar, M., A.R. Abdul-Aziz, S.A. Maideen y S. Z. Mohd, Entrepreneurship in the tourism industry: Issues in developing countries, doi: 10.1016/j.ijhm.2011.01.003, International Journal of Hospitality Management, 30(4), 827-835 (2011)

Kastenholz. E., M.J. Carneiro, C.P. Marques y J. Lima, Understanding and managing the rural tourism experience The case of a historical village in Portugal, doi: 10.1016/j.tmp.2012.08.009, Tourism Management Perspectives, 4, 207-214 (2012)

Koukios, E. M., M. Monteleone, J. T. Carrondo, A. F. Charalambous, E. L. Girio, Hernández y A. Zabaniotou, Targeting sustainable bioeconomy: A new development strategy for Southern European countries. The Manifesto of the European Mezzogiorno, doi: 10.1016/j.jclepro.2017.05.020, Journal of Cleaner Production, 172, 3931-3941 (2018)

La Hora, Vuelta al Imbabura con innovaciones, Ibarra Ecuador (2017)

Manhas, P. S., L. A. Manrai y A. K. Manrai, Role of tourist destination development in building its brand image: A conceptual model, Journal of Economics, doi: 10.1016/j.jefas.2016.01.001, Finance and Administrative Science, 21(40), 25-29 (2016)

Ministerio de Turismo (2018)

Muñoz, M. J., Proyectar/investigar: Método de educación dialógica, aplicado al desarrollo de competencias, en la iniciación al diseño sostenible, doi: 10.4067/S0718-50062009000200004, Formación Universitaria, 2(2), 17-26 (2009)

Muñoz, P. y D. Dimov, The call of the whole in understanding the development of sustainable ventures, doi: 10.1016/j.jbusvent.2014.07.012, Journal of Business Venturing, 30(4), 632-654 (2015)

Nesbitt. L., N. Hotte, S. Barron, J. Cowan y S. R. Sheppard, The social and economic value of cultural ecosystem services provided by urban forests in North America: A review and suggestions for future research, doi: 10.1016/j.ufug.2017.05.005, Urban Forestry \& Urban Greening, 25, 103-111 (2017)

Nunkoo, R., Tourism development and trust in local government, doi: doi.org/10.1016/j.tourman.2014.08.016, Tourism Management, 46, 623-634 (2015)

O'brien, O., J. Cheshire y M. Batty, Mining bicycle sharing data for generating insights into sustainable transport systems, doi: 10.1016/j.jtrangeo.2013.06.007, Journal of Transport Geography, 34, 262-273 (2014)

Pickering, C. M. y S. Rossi, Mountain biking in peri-urban parks: Social factors influencing perceptions of conflicts in three popular National Parks in Australia, doi: 10.1016/j.jort.2016.07.004, Journal of Outdoor, Recreation and Tourism, 15, 71$81(2016)$

Pickering, C., M., Ansong y E. Wallace, Experimental assessment of weed seed attaching to a mountain bike and horse under dry conditions, doi: 10.1016/j.jort.2016.07.005, Journal of Outdoor Recreation and Tourism, 15, 66-70 (2016) 
Pires, S.M., T. Fidélis y T.B. Ramos, Measuring and comparing local sustainable development through common indicators: Constraints and achievements in practice, doi: 10.1016/j.cities.2014.02.003, Cities, 39, 1- 9 (2014)

Qian, C., N., Sasaki, D., Jourdain, S. M. Kimb y P. G. Shivakoti, Local livelihood under different governances of tourism development in China-A case study of Huangshan mountain area, doi: 10.1016/j.tourman.2017.01.006, Tourism Management, 61, 221-233 (2017)

Reimer, J. K. y P. Walter, How do you know it when you see it? Community-based ecotourism in the Cardamom Mountains of southwestern Cambodia, doi: 10.1016/j.tourman.2012.04.002,Tourism Management, 34, 122-132 (2013)

Sauvé, S., S. Bernard y P., Sloan, Environmental sciences, sustainable development and circular economy: Alternative concepts for trans-disciplinary research, doi:10.1016/j.envdev.2015.09.002, Environmental Development, 17, 48-56 (2016)

Sebele, L.S., Community-based tourism ventures, benefits and challenges: Khama rhino sanctuary trust, central district, Botswana, doi: 10.1016/j.tourman.2009.01.005, Tourism management, 31(1), 136-146 (2010)

Snyman, S., Ecotourism joint ventures between the private sector and communities: An updated analysis of the Torra Conservancy and Damaraland Camp partnership, Namibia, doi:10.1016/j.tmp.2012.07.004, Tourism Management Perspectives, 4, 127-135 (2012)

Soto, S., J. C. Briede y M. L. Mora, Sensibilización Ambiental en Educación Básica: Una Experiencia de Aprendizaje para Abordar la Sustentabilidad utilizando el Diseño y la Ciencia Ficción, doi: 10.4067/S0718- 07642017000200016, Información tecnológica, 28(2), 141-152 (2017)

Sportbike, La Vuelta la Imbabura, Ibarra, Ecuador (2018)

Taher, S.H.M., S. A. Jamal, N. Sumarjan y N. Aminudin, Examining the structural relations among hikers' assessment of pull-factors, satisfaction and revisit intentions: The case of mountain tourism in Malaysia, doi: 10.1016/j.jort.2015.11.012, Journal of Outdoor Recreation and Tourism, 12, 82-88 (2015)

Zhang. L., J. Zhang, Z.Y. Duan y D. Bryde, Sustainable bike-sharing systems: characteristics and commonalities across cases in urban China, doi: 10.1016/j.jclepro.2014.04.006, Journal of Cleaner Production, 97, 124-133 (2015) 